\title{
Expanded Thruster Mass Model Incorporating Nested Hall Thrusters
}

\author{
Scott J. Hall, ${ }^{*}$ Benjamin A. Jorns ${ }^{\dagger}$ and Alec D. Gallimore ${ }^{\ddagger}$ \\ University of Michigan, Ann Arbor, MI, 48109, USA \\ and \\ Richard R. Hofer ${ }^{\S}$ \\ Jet Propulsion Laboratory, California Institute of Technology, Pasadena, CA, 91109, USA
}

\begin{abstract}
A mass and cost model for the selection of electric propulsion string size has been expanded to include the effects of nesting the discharge channels in Hall thrusters. The equations for three components were modified: the thruster, the xenon flow system, and the cabling. We developed an analytical expression for thruster specific mass as a function of the number of nested channels, then used that expression to evaluate the specific mass savings provided by an example channel nesting technique. Xenon flow system and cabling equations were modified to scale with number of channels instead of number of thrusters. The updated model was then applied to missions ranging in power from $500 \mathrm{~kW}$ to 1.25 MW for systems containing zero to five redundant thrusters. These results indicate that the example channel nesting would provide 4-7\% system mass savings as compared to a singlechannel thruster. The impact of nested Hall thrusters on system cost is also explored. It is argued that no modification to the original cost model is necessary to capture nested Hall thrusters. Results for 1-channel and 3-channel thrusters demonstrate that the mass savings translate to improved cost savings, ranging from 9-13\% for the example nesting technique. Other potential nesting techniques are also detailed, and their mass and cost savings are explored using the modified model. These results indicate that system mass savings in excess of $8 \%$ and cost savings in excess of $14 \%$ may be possible with alternative channel nesting techniques. Ultimately, nested thrusters push the system mass minimum toward the cost minimum, providing a means for mission planners to more effectively optimize high-power electric propulsion strings for both cost and mass.
\end{abstract}

\section{Nomenclature}

$\begin{array}{ll}A, B, C, I & \text { regression coefficients } \\ \mathcal{A} & \text { area } \\ d & \text { channel diameter } \\ f & \text { unitless weighting factor } \\ \mathcal{I} & \text { current } \\ j & \text { current density } \\ M & \text { mass } \\ N & \text { number of thrusters } \\ P & \text { power } \\ V & \text { voltage } \\ w & \text { channel width }\end{array}$

Greek Symbols

\footnotetext{
*Ph.D. Candidate, Department of Aerospace Engineering, sjhall@umich.edu, Student Member, AIAA.

${ }^{\dagger}$ Assistant Professor, Department of Aerospace Engineering, bjorns@umich.edu, Senior Member, AIAA.

${ }^{\ddagger}$ Robert J. Vlassic Dean of Engineering, Richard F. and Eleanor A. Towner Professor, and Arthur F. Thurnau Professor, Department of Aerospace Engineering, alec.gallimore@umich.edu, Fellow, AIAA.

$\S$ Group Supervisor, Electric Propulsion, richard.r.hofer@jpl.nasa.gov, Associate Fellow, AIAA
} 


$\begin{array}{ll}\Delta z & \text { thruster thickness } \\ \rho & \text { thruster density } \\ \text { Subscripts } & \\ a c & \text { active thruster } \\ \text { cab } & \text { cabling } \\ \text { gim } & \text { gimbal } \\ i & \text { channel number } \\ N H T & \text { Nested Hall Thruster } \\ \text { outer } & \text { outermost channel } \\ r d & \text { redundant thruster } \\ s & \text { structure } \\ \text { str } & \text { string } \\ \text { sys } & \text { system } \\ \text { tank } & \text { tankage } \\ \text { tg } & \text { thruster and gimbal } \\ \text { th } & \text { thruster } \\ \text { tot } & \text { total } \\ X e & \text { xenon } \\ \text { XFS } & \text { xenon flow system }\end{array}$

\section{Introduction}

C LECTRIC propulsion systems at power levels in excess of $100 \mathrm{~kW}$ are enabling for a number of important Emid- and far-term space missions. At power levels of 300-600 kW, potential missions include cargo tugs to near-earth asteroids, ${ }^{1}$ the moons ${ }^{2}$ and surface ${ }^{3}$ of Mars. At power levels of $700 \mathrm{~kW}$ and above, potential missions include human crew capsules to the moons ${ }^{2}$ and surface ${ }^{4}$ of Mars. The increasing availability of solar power on orbit ${ }^{1}$ is improving the feasibility of these mission concepts. As this occurs, more attention is being paid to how to select the makeup of the electric propulsion system. One attractive technology is the Hall thruster, ${ }^{5}$ a device that uses perpendicular electric and magnetic fields to ionize and accelerate propellant. Hall thrusters can provide total efficiencies in excess of $60 \%$ at specific impulses of up to 3500 seconds on xenon propellant and up to 5000 seconds on krypton. Hall thrusters have a long flight heritage, ${ }^{5}$ but this has been limited to individual thruster powers of $4.5 \mathrm{~kW}$ in flight ${ }^{6}$ and $12.5 \mathrm{~kW}$ in near-term planned missions. ${ }^{7}$ Traditional Hall thrusters have been developed at powers in excess of $50 \mathrm{~kW},{ }^{8}$ but these have never extended beyond laboratory demonstration.

Electric propulsion can be applied to missions in a variety of ways ranging from a single thruster at the full system power level to an array of many small lower-power thrusters operating concurrently. The decision on which configuration to select is a trade among a number of factors including system mass, cost, performance,

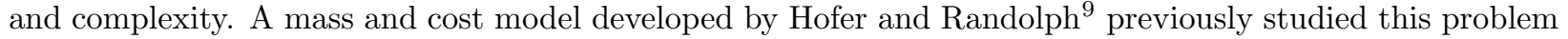
for single-channel Hall thrusters (SHTs). In that work, it was found that for a given system power level, the cost strongly minimized for fewer thrusters, yet the mass demonstrated a weak minimum centered at a larger number of thrusters than the cost minimum. The Hofer-Randolph model featured high fidelity and was able to provide insight from a system-level perspective. However, their results focused only the single-channel Hall thruster. Incorporating other types of electric propulsion devices, including alternative Hall thruster configurations, may optimize the problem differently.

One such alternative configuration is the nested-channel Hall thruster (NHT), wherein Hall thruster discharge channels are concentrically stacked. This technique, which has origins in the Russian literature ${ }^{10}$ was first explored experimentally in two $10-\mathrm{kW}$ class thrusters. ${ }^{11,12,13,14}$ Following the successful development of low-power NHTs, a 100-kW class thruster ${ }^{15,16,17,18}$ known as the X3 was developed by the University of Michigan, the Air Force Research Laboratory, NASA, and ElectroDynamic Applications. The X3 is designed to be capable of operation from 300-800 V discharge voltage and 2-200 kW discharge power. Representative photographs of a SHT, the 20-kW NASA-300M, and a NHT, the X3, are shown in Figure 1. Work done during the development of the X2 and X3 demonstrated the potential for the NHT concept to provide significant savings in device specific mass over a comparably-powered single-channel thruster ${ }^{20,15}$ However, to date these mass savings have remained notional and not been rigorously explored, and the impacts at the 


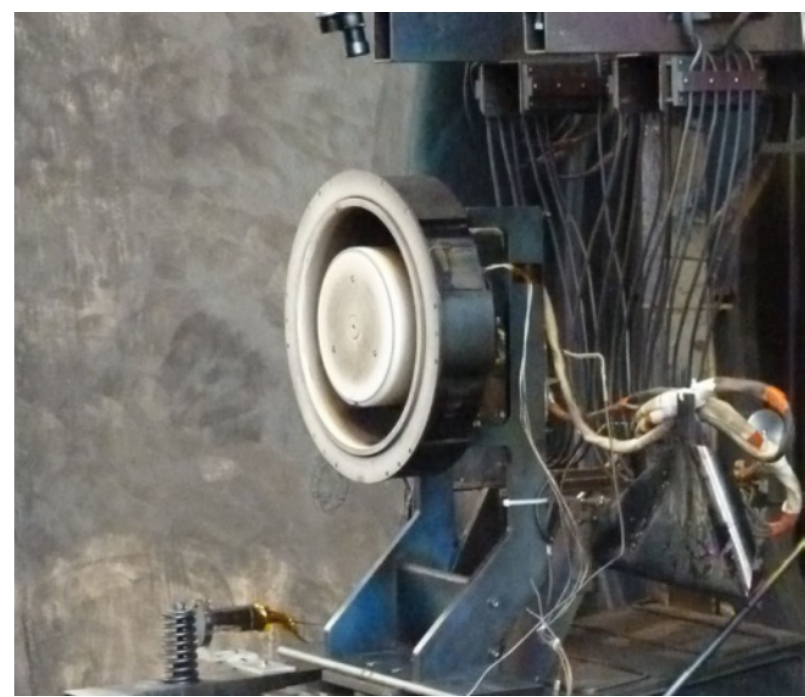

(a) A $20-\mathrm{kW}$ SHT, the NASA-300M. ${ }^{19}$

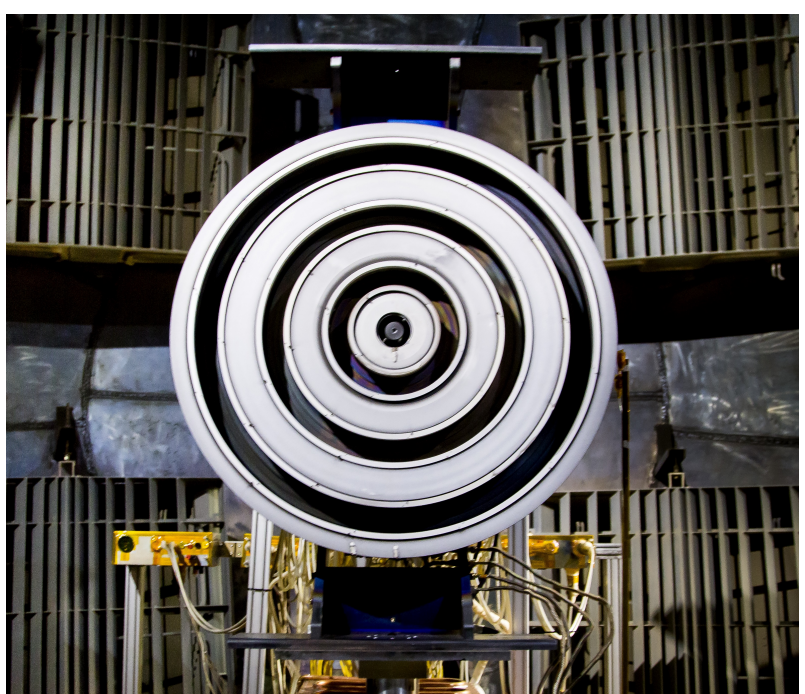

(b) A $200-\mathrm{kW}$ three-channel NHT, the X3. ${ }^{18}$

Figure 1. Representative photographs of single-channel and nested-channel Hall thrusters.

system level have not been studied.

Thus, though work is ongoing to characterize and further develop these devices, there is a need to further quantify the configuration's potential advantages. With appropriate modifications, the Hofer-Randolph model can be leveraged to study this problem. Our work here details the modifications to the HoferRandolph model necessary to capture NHTs and presents results from the model with these changes. This paper is organized as follows. First, a summary of the original Hofer-Randolph model is presented. An explanation and justification of the modifications necessary to apply the model to NHTs is given. Then, a general expression for NHT thruster specific mass is derived. Next, an example NHT nesting technique based off of the X3 thruster is detailed, and the general expression is used to produce thruster specific mass values for it. These thruster specific mass values are then applied to the mass model for missions of $500 \mathrm{~kW}, 750$ $\mathrm{kW}, 1.0 \mathrm{MW}$, and 1.25 MW and varying numbers of redundant thrusters. The cost model is also discussed, and we argue that no modifications are necessary for the model to capture NHTs. Example results from the cost model are then presented. Results are discussed in the context of mission planning and system design, and finally conclusions and potential future refinements of the model are detailed.

\section{Description of Mass Model}

This work expands on a electric propulsion system mass and cost model originally developed by Hofer and Randolph. ${ }^{9}$ Their model was designed to study the optimum number of thrusters for a given total mission power requirement. To do so, the model consisted of two main equations: one for system mass and one for system cost. Though the model was tuned to focus on single-channel Hall thrusters, it was designed to be applicable to any type of electric propulsion technology with appropriate input data. The model captured the entire electric propulsion string, including the thruster, gimbal, xenon flow controller (XFC), propellant management assembly (PMA), power processing unit (PPU), xenon tank, and cabling. An image of the system, reproduced from Hofer and Randolph, ${ }^{9}$ is shown in Figure 2. For a given total mission power requirement, the electric propulsion string can consist of a single high-power thruster or an abundance of lower-power thrusters.

As discussed by Hofer and Randolph, the nature of electric propulsion and its typical failure mechanisms (high-voltage issues including grounding and arcing, as well as thermal issues) have led the community to adopt a fault tolerance scheme involving the inclusion of one, two, or three redundant thruster strings corresponding to single-, double-, or triple-fault tolerance. Single-fault tolerance is typically adequate for science missions without humans on board, whereas double- or triple-fault tolerance may be required for missions involving human crews. ${ }^{9}$ For example, the ion propulsion system for the Dawn mission was in a "2 plus 1" configuration, in which there were two active ion thrusters and one redundant thruster. ${ }^{21}$ The 


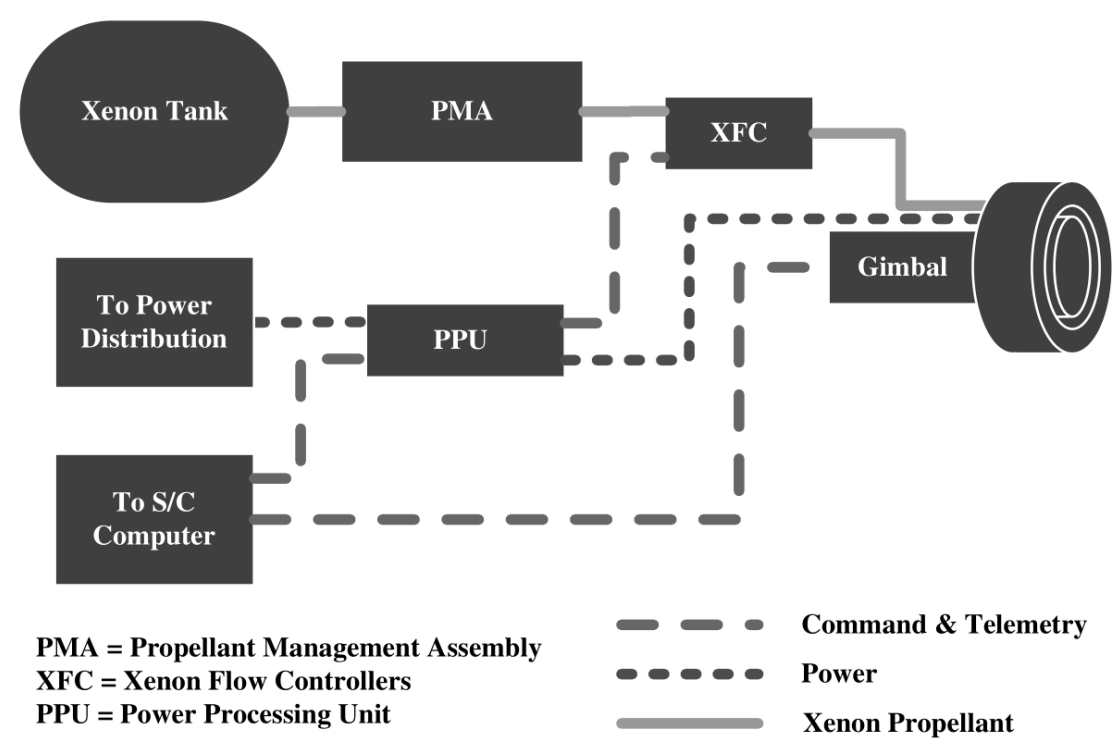

Figure 2. The major elements of a Hall thruster propulsion system. ${ }^{9}$

Hofer-Randolph model can capture an arbitrary fault-tolerance level, which allows for study of the effects of carrying redundant thrusters on system optimization.

The mass equation is a combination of mass estimations of the various system components. Each of these components has a scaling equation that incorporates (typically linear) coefficients established by the authors. These coefficients were gathered from a number of sources, including scientific literature, flight programs, and industry sources. The global mass equation takes the form:

$$
M_{s y s}=\left(1+f_{s}\right)\left(M_{t g}+M_{P P U}+M_{X F S}+M_{c a b}+M_{t a n k}\right),
$$

where $M_{\text {sys }}$ is system mass, $f_{s}$ is the structural weighting factor, $M_{t g}$ is the mass of the thruster and gimbal, $M_{P P U}$ is the mass of the power processing unit, $M_{X F S}$ is the mass of the xenon flow controller, $M_{c a b}$ is the mass of cabling, and $M_{\text {tank }}$ is the mass of the propellant tankage. This can be expanded to:

$$
\begin{array}{r}
M_{\text {sys }}=\left(1+f_{s}\right)\left\{( N _ { t o t } ) \left[\left(A_{t g}+A_{P P U}+A_{c a b}\right)\left(\frac{P_{s y s}}{N_{a c}}\right)+B_{t g}+B_{P P U}\right.\right. \\
\left.\left.+B_{X F S}+B_{c a b}\right]+C_{X F S}+\left(1+f_{\text {tank }}\right) I_{X e} P_{s y s}\right\},
\end{array}
$$

where $A, B, C$, and $I$ are all linear regression coefficients for the various sub-systems, $N_{\text {tot }}$ is the total number of thrusters, $P_{\text {sys }}$ is the total system power, and $N_{a c}$ is the number of active thrusters. The various sub-system equations take the following forms. Thruster and gimbal:

$$
\begin{aligned}
M_{t g} & =M_{t h}+M_{g i m} \\
& =\left(1+f_{\text {gim }} D_{g i m}\right)\left(N_{a c}+N_{r d}\right)\left[A_{t h}\left(\frac{P_{s y s}}{N_{a c}}\right)+B_{t h}\right] \\
& =\left(N_{a c}+N_{r d}\right)\left[A_{t g}\left(\frac{P_{s y s}}{N_{a c}}\right)+B_{t g}\right],
\end{aligned}
$$

where $M_{t h}$ is the mass of the thruster, $M_{g i m}$ is the mass of the gimbal, $D$ is a regression coefficient, and $N_{r d}$ is the number of redundant thrusters. Power processing unit:

$$
M_{P P U}=\left(N_{a c}+N_{r d}\right)\left[A_{P P U}\left(\frac{P_{s y s}}{N_{a c}}\right)+B_{P P U}\right],
$$


cabling:

$$
M_{c a b}=\left(N_{a c}+N_{r d}\right)\left[A_{c a b}\left(\frac{P_{s y s}}{N_{a c}}\right)+B_{c a b}\right]
$$

xenon flow system:

$$
M_{X F S}=\left(N_{a c}+N_{r d}\right) B_{X F S}+C_{X F S}
$$

xenon propellant:

$$
M_{X e}=I_{X e} P_{\text {sys }}
$$

and tankage:

$$
\begin{aligned}
M_{\text {tank }} & =f_{\text {tank }} M_{X e} \\
& =f_{\text {tank }} I_{X e} P_{\text {sys }} .
\end{aligned}
$$

For each of the subsystem components above, linear scaling is used as a first order approximation. Subsystems either scale with the power of each thruster (thruster, PPU), the number of strings (XFS, cabling), the total system power (xenon), or the total propellant mass (tankage). Except for the changes detailed below, our work here used the same nominal coefficients used by Hofer and Randolph. For reference, these coefficients are presented in Table 1.

\begin{tabular}{c|cccccc}
$\begin{array}{c}\text { Name } \\
\text { (subscript) }\end{array}$ & $\begin{array}{c}\mathrm{A} \\
{[\mathrm{kW} / \mathrm{kg} / \text { string }]}\end{array}$ & $\begin{array}{c}\mathrm{B} \\
{[\mathrm{kg} / \mathrm{string}]}\end{array}$ & $\begin{array}{c}\mathrm{C} \\
{[\mathrm{kg}]}\end{array}$ & $\begin{array}{c}\mathrm{D} \\
{[-]}\end{array}$ & $\begin{array}{c}\mathrm{f} \\
{[-]}\end{array}$ & $\begin{array}{c}\mathrm{I} \\
{[\mathrm{kg} / \mathrm{kW}]}\end{array}$ \\
\hline \hline Thruster (th) & Variable & 0 & - & - & - & - \\
Gimbal (gim) & - & - & - & 0.5 & - & - \\
PPU (ppu) & 1.7419 & 4.654 & - & - & - & - \\
XFS (xfs) & - & 3.2412 & 4.5189 & - & - & - \\
Cabling (cab) & 0.06778 & 0.7301 & - & - & - & - \\
Structural (s) & - & - & - & - & 0.26 & - \\
Tankage (tank) & - & - & - & - & 0.04 & - \\
Xenon (Xe) & - & - & - & - & - & 100
\end{tabular}

Table 1. Mass model coefficients used in this work. These values come from the work of Hofer and Randolph. ${ }^{9}$

It is important to note that Hofer and Randolph's thruster specific mass coefficient, $A_{t h}$, was determined by fitting a line to a set of masses and nominal powers for single-channel Hall thrusters. By doing so, the authors implicitly impose certain thruster characteristics: namely, nominal Hall thruster current densities and discharge voltages of 300-400 V. To appropriately compare results, these characteristics must be imposed on the NHT designs below as well. This puts arbitrary limits on thrusters and does not capture the entire performance envelope of Hall thrusters. To use the X3 as an example, at $300 \mathrm{~V}$ and a nominal discharge current density the X3 is an $80 \mathrm{~kW}$ thruster, implying that $3 \mathrm{X} 3 \mathrm{~s}$ would be necessary for a $200-\mathrm{kW}$ mission. Yet the X3 has a 200-kW throttle point at $800 \mathrm{~V}$ discharge voltage, meaning that a single X3 has the potential to serve the needs of a $200-\mathrm{kW}$ mission.

\section{Modifications to Mass Model to Capture NHTs}

There are three terms in the Hofer-Randolph model that we change to accommodate NHTs: the cabling, xenon flow system, and thruster specific mass. The cabling and XFS terms are modified such that they scale per channel, not per thruster, and the thruster specific mass is modified to vary with the number of channels. The PPU equation is not changed because the discharge units for high-power SHTs (the source of the bulk of the mass and cost in the PPU) are likely to be made up of lower-power $(10-20 \mathrm{~kW})$ sub-units. For a NHT at the same power, these would simply be distributed across the $N_{c h}$ channels. The cabling required by this 
is captured in the $M_{c a b}$ term. It is expected that there will be a small increase in PPU mass with increasing number of channels due to the fact that each channel requires its own magnet power supplies, but these will remain less than $1 \mathrm{~kW}$ each and thus a small fraction of the discharge power. Additionally, because NHTs are capable of operating off of a single centrally-mounted cathode, ${ }^{14,16}$ the cathode heater and keeper requirements will be identical for a given thruster power level, regardless of the number of channels. Thus, we do not modify the PPU mass scaling.

\section{A. Cabling and XFS}

The equations for cabling and the xenon flow system are modified such that they are multiplied by the number of channels in each thruster $\left(N_{c h}\right)$ :

$$
M_{c a b}=N_{c h}\left(N_{a c}+N_{r d}\right)\left[A_{c a b}\left(\frac{P_{s y s}}{N_{a c}}\right)+B_{c a b}\right] \text {, }
$$

and

$$
M_{X F S}=N_{c h}\left(N_{a c}+N_{r d}\right) B_{X F S}+C_{X F S}
$$

This accounts for the fact that each active channel requires cabling and xenon mass flow control. With these modifications, the global mass model equation becomes:

$$
\begin{array}{r}
M_{s y s}=\left(1+f_{s}\right)\left\{( N _ { t o t } ) \left[\left(A_{t g}+A_{P P U}+N_{c h} A_{c a b}\right)\left(\frac{P_{s y s}}{N_{a c}}\right)+B_{t g}+B_{P P U}\right.\right. \\
\left.\left.+N_{c h} B_{X F S}+B_{c a b}\right]+C_{X F S}+\left(1+f_{t a n k}\right) I_{X e} P_{s y s}\right\} .
\end{array}
$$

\section{B. Thruster Specific Mass}

\section{General Expression}

For an NHT, we denote total thruster power as the sum of the discharge powers of each channel:

$$
P_{t h}=\sum_{i=1}^{N_{c h}} V_{D, i} \mathcal{I}_{i}
$$

where $V_{D, i}$ is the discharge voltage, and $\mathcal{I}_{i}$ is the current for the $i^{\text {th }}$ channel. The current for each channel can be expressed as

$$
\mathcal{I}_{i}=j_{i} \mathcal{A}_{i}
$$

where $j_{i}$ is current density and $\mathcal{A}_{i}$ the exit area of the $i^{\text {th }}$ channel. A Hall thruster channel is an annulus, the area of which is

$$
\mathcal{A}_{i}=\pi d_{i} w_{i}
$$

where the characteristic dimensions of the channel are the mean diameter $d_{i}$ and the channel width $w_{i}$. Equations 14 and 13 then can be substituted into Equation 12 to yield

$$
P_{t h}=\pi \sum_{i=1}^{N_{c h}} V_{D, i} j_{i} d_{i} w_{i}
$$

Thruster mass can be expressed as:

$$
m=\rho \pi\left(\frac{d_{N_{c h}}}{2}+\frac{w_{N_{c h}}+w_{p o l e, N_{c h}}}{2}\right)^{2} \Delta z
$$


where $\rho$ is the thruster volumetric mass density, $d_{N_{c h}}$ is the diameter of the outermost channel, $w_{N_{c h}}$ is the width of the outermost channel, $w_{p o l e, N_{c h}}$ is the width of the outer channel's poles, and $\Delta z$ is the thickness of the thruster. This expression becomes

$$
m=\frac{\pi}{4} \rho \Delta z\left(d_{N_{c h}}+w_{N_{c h}}+w_{p o l e, N_{c h}}\right)^{2} .
$$

Equations 15 and 17 then can be combined to create an expression for the NHT thruster specific mass:

$$
A_{t h, N H T}=\frac{\rho \Delta z\left(d_{N_{c h}}+w_{N_{c h}}+w_{p o l e, N_{c h}}\right)^{2}}{4 \sum_{i=1}^{N_{c h}} V_{D, i} j_{i} d_{i} w_{i}} .
$$

Equation 18 serves as a general analytical expression for the NHT geometry with an arbitrary number of channels, where no particular thruster design assumptions have been made.

\section{Example NHT Nesting Technique}

A set of thruster design assumptions can be applied to Equation 18 to provide quantitative values of $A_{t h, N H T}$ for use in the mass model. As discussed above, a discharge voltage of $300 \mathrm{~V}$ and a typical Hall thruster current density must be selected such that comparisons to the results of the original work are valid. This discharge voltage and current density is assumed to be constant across all channels and can be pulled outside of the summation in Equation 18. Next, we design an example NHT channel nesting technique. First, we assume that the thruster front magnetic pole pieces, which must be accounted for to scale the channels such that they do not overlap, are approximately one-half the width of the channel to which they are adjacent. This is seen as a reasonable approximation: Hall thruster scaling work has demonstrated that maintaining appropriate magnetic field strengths on channel centerline is key to proper thruster operation, ${ }^{22}$ a task that will require more magnetic circuit material as channel width increases. Thus, as a first-order approximation, channel width and pole piece size scale together. Additionally, it is assumed that pole pieces between nested channels are shared but are twice as wide. This approximately agrees with the designs of both the X2 and the X3.

There are many possible schemes for nesting the channels of an NHT. Thruster power goes with discharge channel exit area, so a design goal to maximize the benefit of the NHT configuration is to maximize the amount of exit area contained within a given thruster outer diameter. However, the magnetic pole pieces inhibit this. Ultimately the nesting technique selected must be balance engineering practicality with optimized geometry. The X3 provides one such solution. The nesting technique used in the X3 features channel widths that increase slightly with increasing channel diameter, of the general form:

$$
w_{i}=w_{1}+\alpha(i-1),
$$

where $w_{i}$ is the width of the $i^{\text {th }}$ channel, $w_{1}$ is width of the innermost $(i=1)$ channel, and $\alpha$ is a scale factor. For this example nesting technique, $\alpha$ is set to one.

The remaining free variables in Equation 18 are the thruster density, $\rho$, and the thruster thickness $\Delta z$. Hall thruster scaling work has shown that the discharge channel length (and thus thruster thickness) are primarily dictated by the discharge voltage, ${ }^{22}$ which is held at a constant $300 \mathrm{~V}$. Thus, $\Delta z$ is simply combined with thruster density $\rho$, and a value for $\rho \Delta z$ is chosen such that the Hofer and Randolph single-channel $A_{t h}$ value of 2.425 is recovered for single-channel thrusters. This assumes that the value of $\rho \Delta z$ remains constant no matter how the channels are scaled.

The values for $A_{t h, N H T}$ produced by this nesting technique in Equation 18 are presented in Table 2.

\begin{tabular}{c|c}
$N_{c h}$ & $A_{t h, N H T}$ \\
\hline 1 & 2.425 \\
2 & 1.941 \\
3 & 1.851 \\
4 & 1.841 \\
5 & 1.854
\end{tabular}

Table 2. Values for $A_{t h, N H T}$ from Equation 18 for the example NHT channel nesting technique. 
It is worth noting that this particular nesting technique demonstrates asymptotic behavior for $N_{c h} \geq 3$. This indicates that for this method the benefits from nesting more than three channels may be limited. The implications of this at the system level are explored below.

It can be argued that the NHT configuration only becomes useful at large enough thruster sizes, i.e., high enough thruster powers, that significant diameter savings can be achieved by nesting discharge channels. Single-channel Hall thrusters have been successfully demonstrated up to power levels of $50-\mathrm{kW},{ }^{8}$ so this power level is chosen as an lower limit for NHT application.

\section{Mass Model Results}

We now use the values of $A_{t h, N H T}$ shown in Table 2 in Equation 11, and apply it to four high-systempower mission profiles: $500 \mathrm{~kW}, 750 \mathrm{~kW}, 1.0 \mathrm{MW}$, and $1.25 \mathrm{MW}$. These results are intended to demonstrate the basic trends of NHTs as compared to single-channel thrusters and the potential advantages as system power level increases. The plots presented below are designed to provide direct comparison to the results found in the original Hofer-Randolph model. For a given total system power level, the model calculates the expected system mass for $N_{a c}$ number of active thrusters (plotted along the abscissa in the following figures). Thus, for a 500-kW system power, "1 active thruster" corresponds to a system of one active and one redundant thruster at input powers of $500 \mathrm{~kW}$ each, "2 active thrusters" corresponds to a system of two active and one redundant thrusters at input powers of $250 \mathrm{~kW}$ each, and so on. To facilitate direct comparisons, the results are normalized against the minimum value of the $N_{c h}=1$ case. Thus, values from the ordinate of the plots correspond to the relative mass as compared to the single-channel minimum value.

\section{A. Variation with System Power Level}

Figure 3 presents the results for X3-like scaling for a 1-MW system with one redundant thruster. The profiles are plotted as the normalized system mass versus the number of active thrusters. All curves display a broad

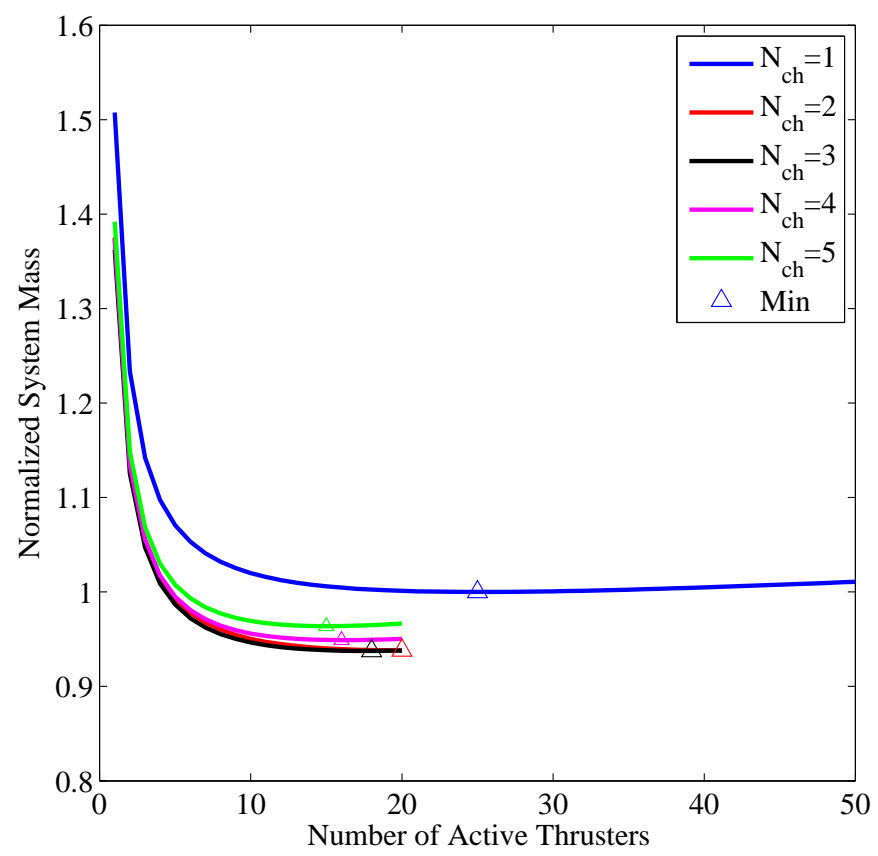

Figure 3. System mass profile for X3-like NHT scaling for values of $N_{c h}=1-5$ and 1 redundant thruster at 1-MW system power. The minimum of each curve is denoted with a triangle.

plateau near the minimum, similar to the original Hofer-Randolph model results. The original work notes that there were wide ranges of thruster powers (and thus number of thrusters) where the system mass is 
within 5 to $10 \%$ of the minimum value depending on system power, giving system designers a large design space in which to work. This result appears in the NHT results as well.

Figure 4 plots the savings at mass minimum versus number of nested channels. We find that the mass

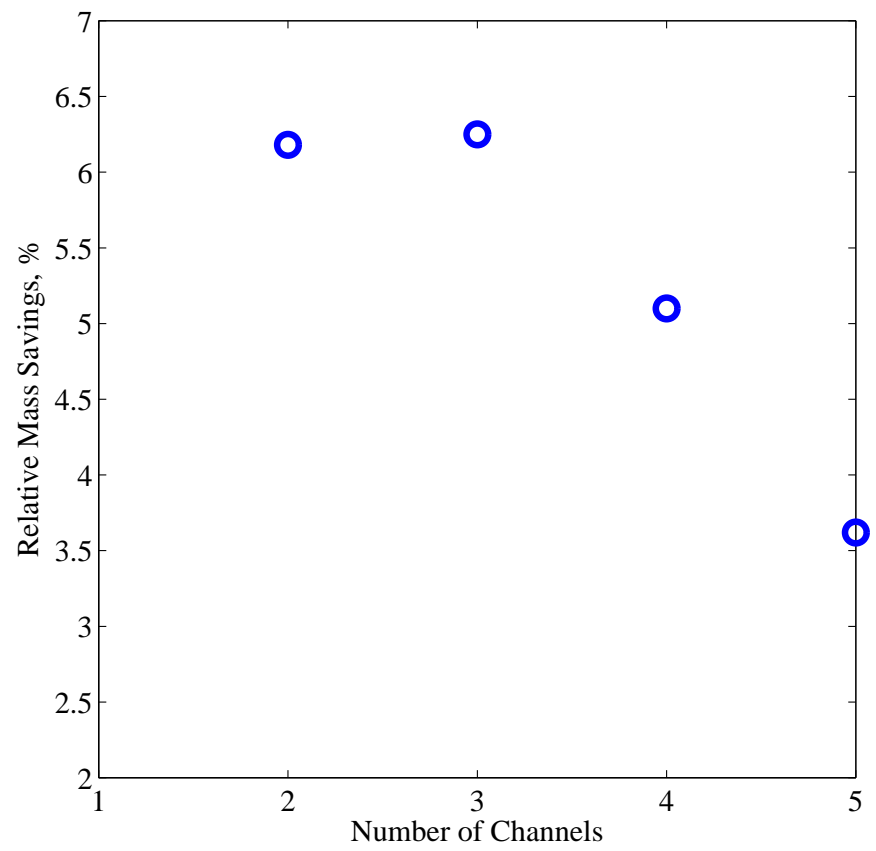

Figure 4. The maximum mass savings for values of $N_{c h}$ between 2 and 5 for a 1-MW mission with X3-like channel nesting.

savings are nearly identical for $N_{c h}=2$ and 3. This result seems odd because Table 2 shows three-channel NHTs to be lighter than two-channel ones with this type of nesting. However, these results can be explained by Figure 5. Here, the thruster mass and cabling/XFS mass are plotted separately along with their sum. It can be seen that the mass savings provided by the lighter thruster in the three-channel case are almost exactly canceled out by the increase in cabling and XFS required by the additional channel. This explains the heavier four- and five-channel systems as well: as shown in Table 2, thruster specific mass is nearly constant for $N_{c h}=3-5$, so the additional cabling and XFS cause these thruster systems to be heavier than the three-channel systems.

Next, we look at results for system powers from $500 \mathrm{~kW}$ to $1.25 \mathrm{MW}$. Figure 6 presents the mass savings provided by nesting $2-5$ channels with the X3-like technique. The trends seen for the 1-MW case are present at all power levels studied. For this nesting technique and a $500 \mathrm{~kW}$ system, 2- and 3-channel thrusters provide approximately $5.5 \%$ savings, and when system power is increased to $1.25 \mathrm{MW}$, mass savings increase to about $6.3 \%$. One outcome of these results that is relevant to the cost model is the fact that the NHT mass minimum typically occurs at smaller number of active thrusters than for SHTs. This result is demonstrated in the plot in Figure 6b. Here, it can be seen that NHTs optimize to anywhere between 5 and 11 fewer thrusters than SHTs. The implications of this result on system cost will be discussed below.

\section{B. Variation with Number of Redundant Thrusters}

The results of the original mass model showed that the mass-optimum number of thrusters for a given system power grew as the number of redundant thrusters increased. This is to be expected: for the extreme case of a single active thruster, a triple-fault-tolerance requirement would require flying one active and three redundant thrusters. This would double the system mass over the single-fault-tolerance (one active and one redundant thruster) case.

Our results showed similar trends, as presented in Figure 7 for a 1-MW system and up to five redundant 


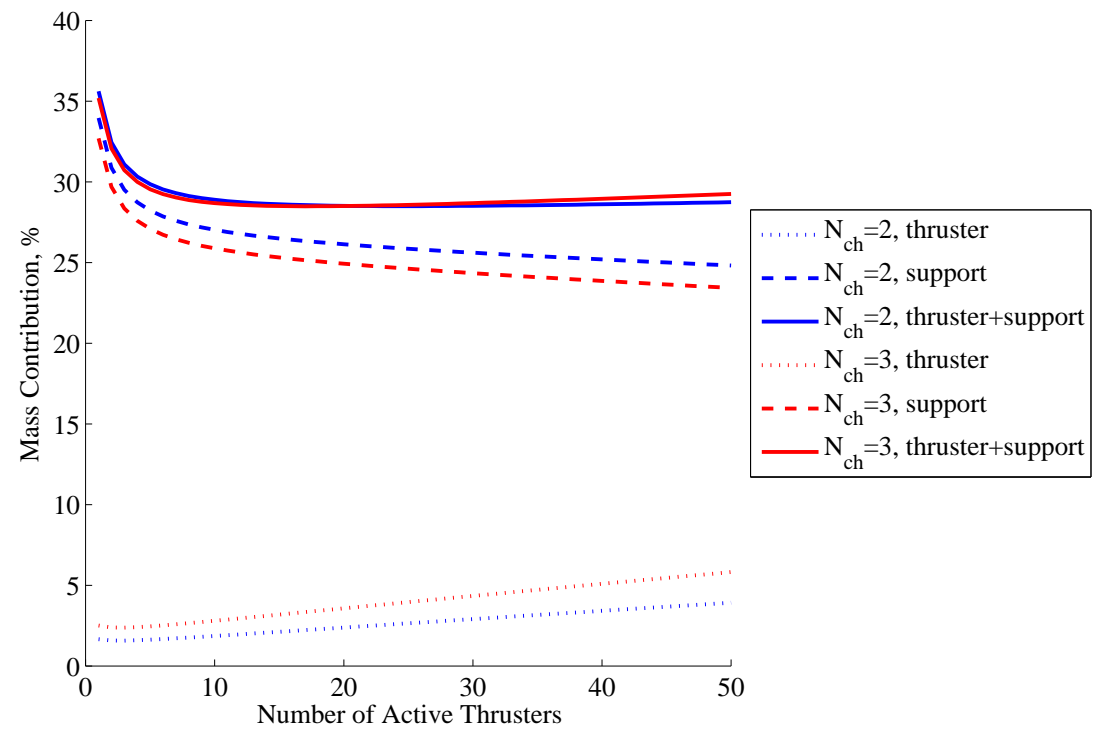

Figure 5. The NHT-modified terms in the mass model plotted separately and summed. The thruster mass savings realized by going from two to three nested channels (dotted lines) are nearly perfectly canceled out by the increase in cabling and XFS required by the additional channel ("support", dashed lines), as evidenced by their sums (solid lines) lying on top of each other.

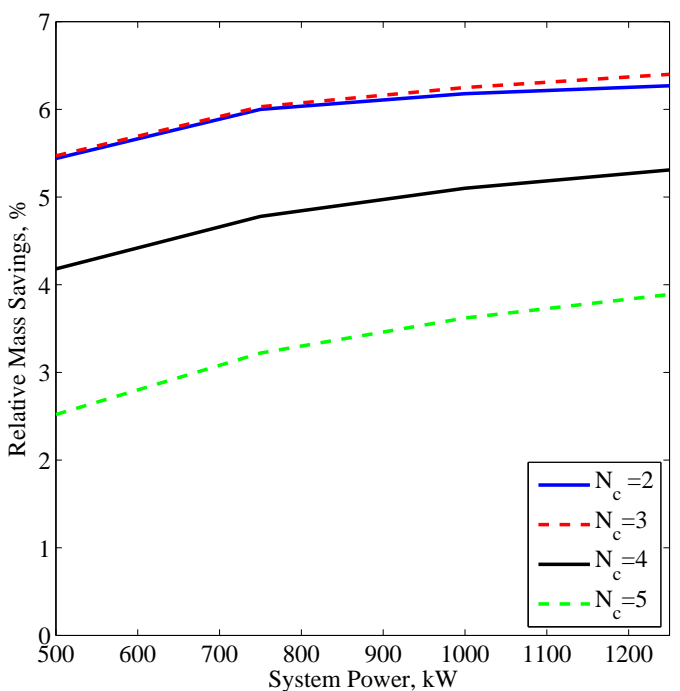

(a) Maximum mass savings

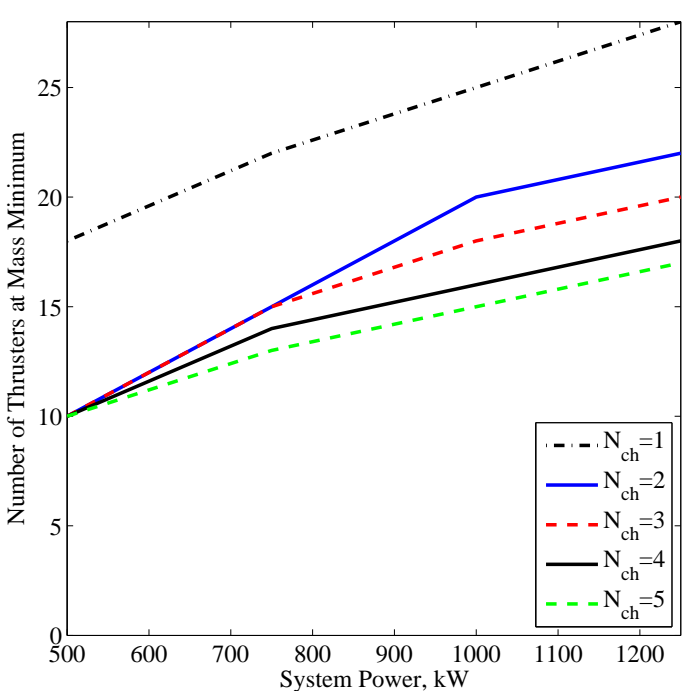

(b) Number of thrusters at mass minimum

Figure 6. The savings provided by NHTs scaled like the X3 for total mission powers of $500 \mathrm{~kW}-1.25 \mathrm{MW}$. These savings manifest both as decreased mass and as fewer number of thrusters. 
thrusters. These results demonstrate a decrease of mass savings with more redundant thrusters. This is

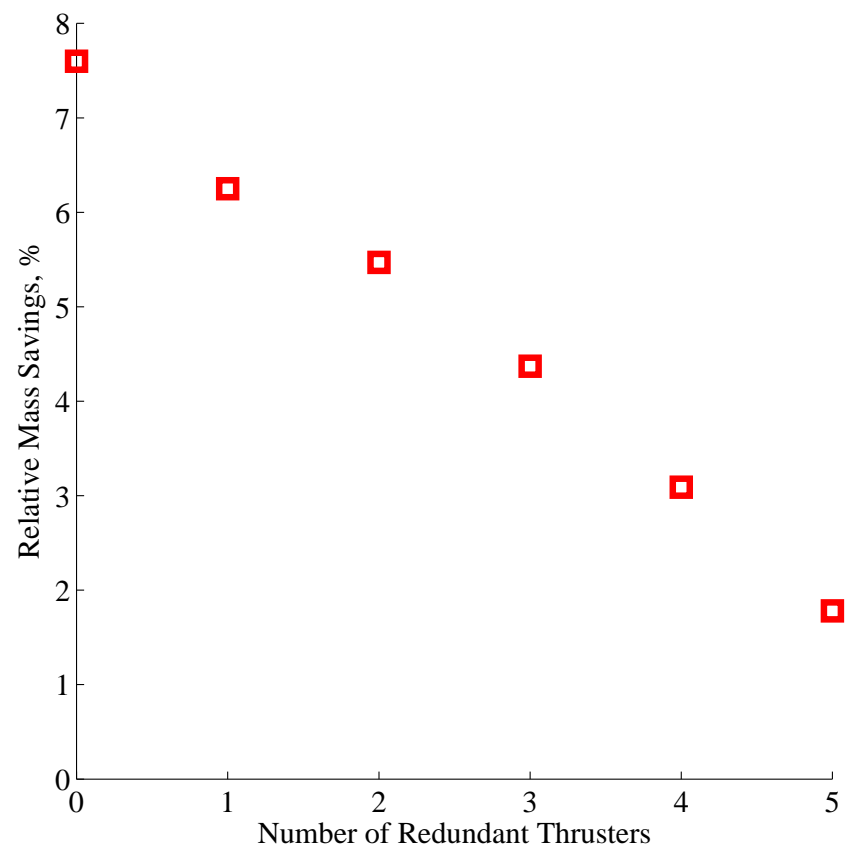

Figure 7. The relative mass savings versus number of redundant thrusters for a 1-MW system with 3 nested channels.

due to the fact that increased redundancy pushes the mass minimum to lower power thrusters (and thus larger numbers of active thrusters, away from the realm of NHTs). As $N_{\text {red }}$ increases from 1 to 3 , the mass savings provided by the NHT geometry decreases from $6 \%$ to $4 \%$. However, the minimum for single-channel thrusters shifts to higher numbers of active thrusters, occurring at 44 active thrusters for $N_{r e d}=3$. This shift to more thrusters has an impact on the cost side of the model, as will be discussed below.

\section{Variation with Number of Active Channels}

Complexity is a factor not captured explicitly by this model, yet complexity adds system risk and thus cost potential due to failures or slips in timeline. Though it is difficult to add the quantification of complexity or risk to the model, one way to explore this is to present the results not as functions of the number of active thrusters but as functions of the number of active channels. This will not fully address the issue, as the question of whether, for instance, a system of five 1-channel thrusters is more complex (more thrusters to manufacture and mount; likely a higher total part count; five cathodes) or less complex (lower power levels lend to easier ground testing; larger thrusters are likely more difficult to manufacture and assemble) than a 5-channel NHT is best answered only with specific thruster designs. However, it presents the results in a unique way and further illustrates the benefits of the NHT configuration.

Results for a 1-MW mission are re-plotted in Figure 8 against the number of active channels instead of the number of active thrusters. Two cases are plotted. First, to isolate the NHT benefit, the relative system masses are plotted for zero redundant thrusters. If number of channels is viewed as an approximation of system complexity, it can be seen that for a given system complexity, the NHT configuration offers notable mass savings, practically regardless of the number of channels being nested. Plotting in this manner removes the effects of the cabling and xenon flow system (because these items contribute equally to equal numbers of channels) and isolates the thruster-based mass savings. Ultimately the mass savings for a given number of active channels is driven by the value of $A_{t h}$, and for $N_{c h}=2-5$ these values are very close together.

Next, the results are presented for one redundant thruster to provide a practical result, as shown in Figure 8-b. Because each redundant thruster is equivalent to $N_{c h}$ redundant channels, NHTs (especially 


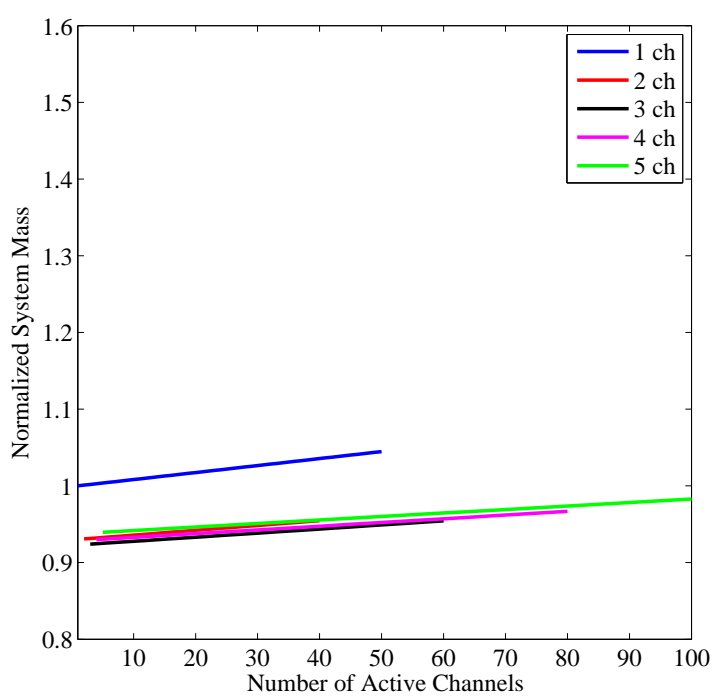

(a) Zero redundant thrusters

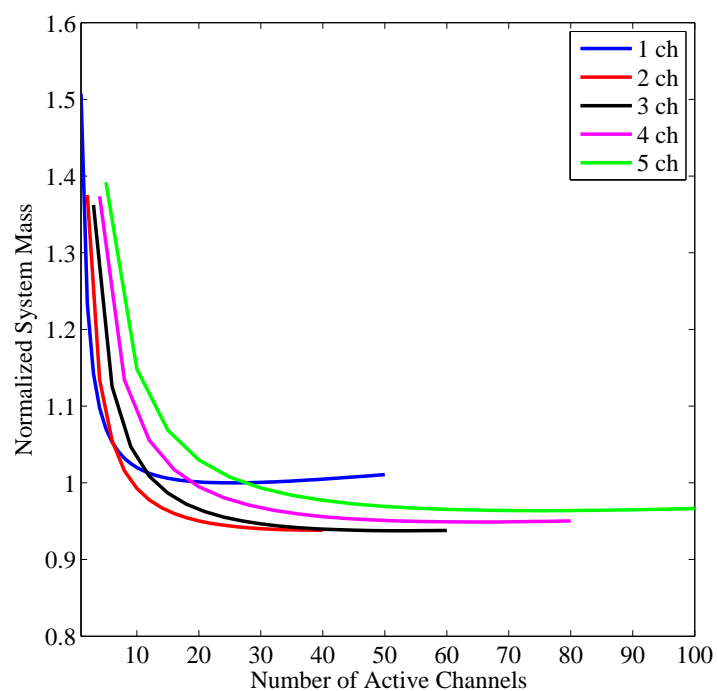

(b) One redundant thruster

Figure 8. Mass model results for X3-like NHT scaling applied to a 1.0-MW system power-level mission for 0 and 1 redundant thrusters, plotted as a function of the total number of active channels instead of the total number of active thrusters. These results illustrate the packing efficiency of the NHT geometry.

those with many channels) see a sharp increase in system mass at very small numbers of channels. At large enough values of active channels, the redundant channels no longer contribute significantly to total system mass, and the increased packing efficiency offered by the NHT configuration becomes clear as in Figure 8-a.

\section{Cost Model}

\section{A. Modifications to Capture NHTs}

The cost portion of the original model splits the system cost into two portions: recurring expenses (which occur for every thruster manufactured in the system) and non-recurring expenses (which occur once for the entire system, regardless of the number of thrusters). Recurring expenses include the cost of raw materials and manufacturing of all of the various string components (thruster, gimbal, PPU, cabling, XFS). Nonrecurring expenses include the string development costs (such as the flight qualification of the thruster). In general, the cost model is not focused on specific dollar amounts but is intended to provide rough-order-ofmagnitude comparisons between system configurations.

A major driver of the recurring expense costs come from the PPU. For the same reasons that the mass is not expected to change significantly, we do not expect the cost to vary significantly between SHTs and NHTs. The manufacturing costs of a single NHT is likely higher than a single-channel thruster of equal power. However, as demonstrated in the mass portion above, an NHT will be lighter. The thruster mass is a good proxy for the cost of raw materials, and as such, it can then be expected that the NHT geometry will offer a savings in raw material cost over single-channel thrusters. To the level of accuracy and specificity of the model, these details are considered likely to balance each other. Finally, though the costs involved with flight-qualifying a high-power thruster are likely quite high due to the increased xenon throughput and thermal loading to the vacuum facility (causing increased usage of liquid nitrogen in cryogenic pumps, for instance), there is no reason to expect these costs to differ between a single-channel thruster and an NHT of the same power. The Hofer-Randolph cost model already factors in these expenses when scaling to high power, and no differentiation between single-channel and multi-channel thrusters of the same power level is necessary.

Due to the fact that the PPU and thruster costs are likely to not vary much between a single-channel and multi-channel Hall thruster at a fixed thruster power level and that flight-qualification costs will be 
the same per $\mathrm{kW}$ between SHTs and NHTs, no attempt is made to modify the cost model here to account for NHTs. However, because the mass and cost are coupled via launch costs, the cost model will produce different curves for NHTs than for SHTs. The differences are explored below.

\section{B. Cost Model Results}

Example results from the cost model are presented in Figure 9 for a 1-MW mission with a single redundant thruster. For clarity, we only present results for $N_{c h}=1$ and 3. The location of the mass minimum of each curve is denoted with a triangle. Just as was shown with the original Hofer-Randolph model, these cost

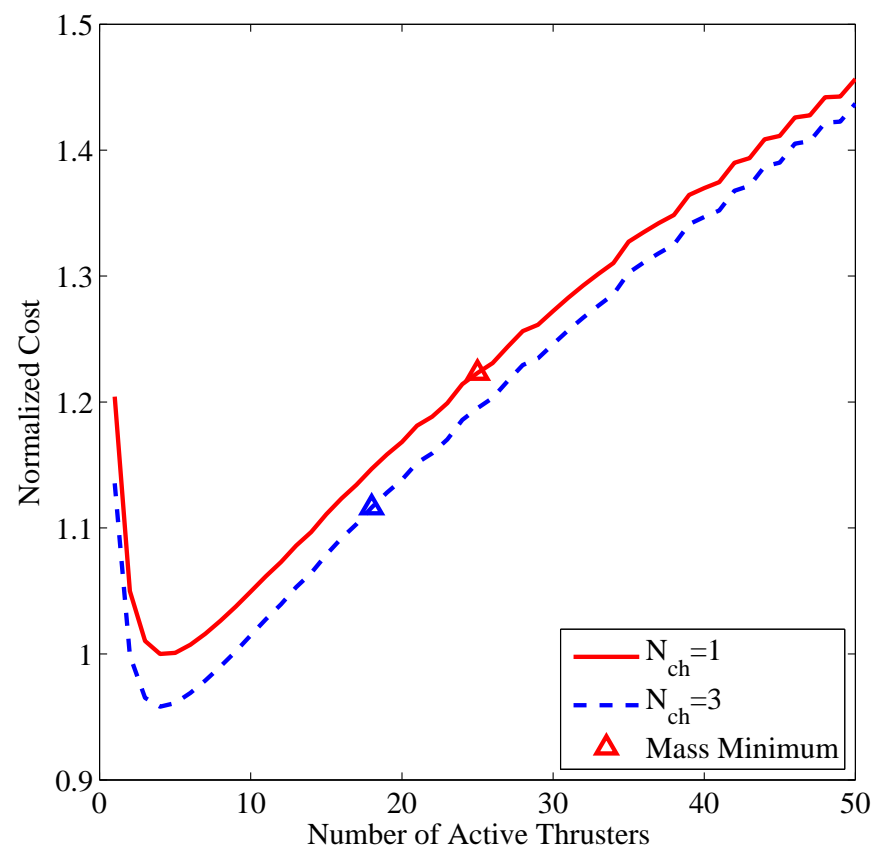

Figure 9. Cost model results for a 1-MW mission with one redundant thruster. The location of the mass minimum is indicated on each curve with a triangle.

curves feature sharp increases on either side of their minima, which are located at values less than 10 active thrusters. The savings in mass provided by NHTs only contributes to a portion of the cost savings, which is represented by the offset between the two lines. This comes from the fact that system mass is directly tied to cost through launch costs; lighter systems are less expensive to launch into orbit, creating the offset between the curves. The other contribution comes from the mass minimum shifting to fewer active thrusters for NHTs. For the 1-MW example mission, the mass minimum shifts from 25 active 1-channel thrusters to 18 active 3-channel thrusters, and this contributes to a further decrease in system cost. In total, for the 1-MW mission, the mass-optimized 3-channel NHT system offers cost savings of approximately $9 \%$ over the mass-optimized 1-channel thruster system.

Next, we calculate the relative cost savings of a 3-channel NHT system as compared to a 1-channel system for mission powers of $500 \mathrm{~kW}$ to $1.25 \mathrm{MW}$. The cost savings offered by the NHT configuration are actually higher for smaller system power levels, even though the relative mass savings are lower. This is due to the fact that at lower powers, NHTs shift the mass minimum farther than for higher power systems, contributing to larger cost savings. For X3-like channel scaling, potential system cost savings predicted by the model range from $9-13 \%$. Savings of this magnitude have the potential of equaling several $\$ 10$ s of million or more. 


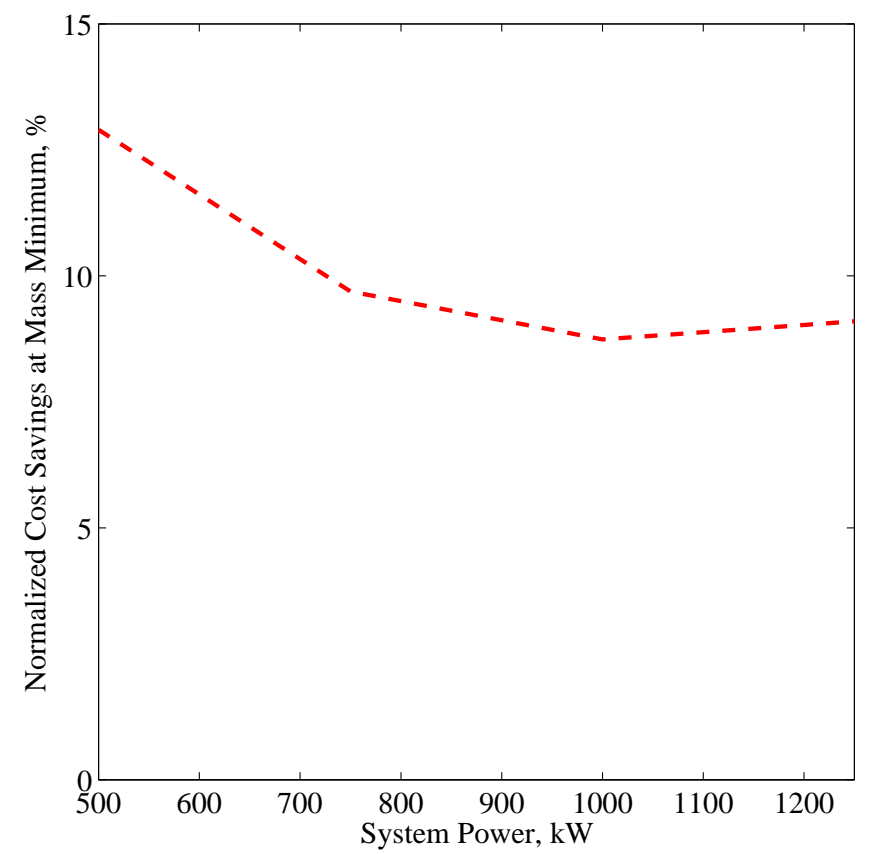

Figure 10. Relative cost savings of a 3-channel NHT system as compared to a 1-channel system versus total system power.

\section{Discussion}

Our results indicate that the impact on overall propulsion system mass provided by the reduced thruster specific mass of an NHT geometry similar to the X3 NHT is small but not negligible. This can be attributed to the fact that the thruster mass itself makes up less than $40 \%$ of the total system mass in the Hofer-Randolph model. Placing the potential impact of thruster mass savings in the context of the entire propulsion system is important from both a mission planning perspective and a thruster development perspective. A 3-channel NHT nested like the X3 produces a thruster that is approximately $24 \%$ lighter than a SHT of the same power level. Yet when compared to an optimized system of SHTs (for a 1-MW system, 28 SHTs), the same 3 -channel NHT only offers total propulsion system mass savings on the order of $6 \%$. The modest mass savings correspond to slightly higher savings in cost, driven by the combination of the improved mass and the shift in mass-optimized number of thrusters to a smaller value. X3-like nesting provides cost savings ranging from $9-13 \%$ for 3 -channel thrusters at mass minimum.

However, this X3-like nesting technique is not the only method to nest channels. Other potential scaling options include constant-width channels and channel widths that scale at constant $w / d$. The limiting case, where there are infinitely-thin magnetic pole pieces, is not a physical thruster design solution but provides an upper bound of the available mass savings. To explore these options, we calculate $A_{t h, N H T}$ for the following nesting techniques:

1. Constant $w$ : For this example geometry, the innermost channel is designed using a fixed $w / d$ ratio, and the remaining channels are also $w_{1}$ wide:

$$
w_{i}=w_{1}
$$

This results in channels that are equally spaced with equal-sized magnetic pole pieces between them.

2. Constant $w / d$ : Here, each successive channel is wider at a constant ratio of $w / d$. This scaling agrees with some literature on single-channel thrusters. ${ }^{23}$ Here, the same $w / d$ value from above is used for 
all 5 thruster channels, which results in channels that get successively farther apart:

$$
w_{i}=\left(\frac{w}{d}\right) d_{i}
$$

3. Infinitely Thin Pole Pieces: This is the limiting case where the entire thruster face consists of exit area and there are in essence no distinct channels. This method will provide an upper bound for the NHT configuration.

The values of $A_{t h, N H T}$ produced by these scaling methods are presented in Table 3 .

\begin{tabular}{c|ccc}
$N_{c h}$ & Constant w & Constant w/d & Infinitely Thin Poles \\
\hline 1 & 2.425 & 2.425 & 2.425 \\
2 & 2.160 & 1.828 & 1.676 \\
3 & 2.110 & 1.692 & 1.440 \\
4 & 2.100 & 1.652 & 1.329 \\
5 & 2.101 & 1.639 & 1.266
\end{tabular}

Table 3. Values of $A_{t h, N H T}$ as calculated by Equation 18 for three alternative channel nesting techniques. All are for $300 \mathrm{~V}$ operation at nominal Hall thruster current densities.

In Figure 11 we compare the mass savings offered by these alternative nesting techniques. These results match what would be expected from the values given in Table 2 . The Infinitely Thin Pole scaling provides the greatest mass savings because it provides the lowest values of $A_{t h, N H T}$; the Constant $w$ scaling provides minimal savings due to its modest decreases in $A_{t h, N H T}$. The curves also help to illustrate the effects of the asymptotic nature of the $A_{t h, N H T}$ values: for the Constant $w$ scaling, where $A_{t h, N H T}$ values for $N_{c h}=3-5$ are all approximately equal, the three-channel geometry actually provides smaller system masses than the two-channel; for the Constant $w / d$ method, where the values of $A_{t h, N H T}$ have not plateaued fully even at $N_{c h}=5$, the three-channel geometry still offers mass savings in excess of $1.5 \%$ over the two-channel.

These mass savings correspond to cost savings in the same manner as for X3-like nesting. Cost model results are presented in Figure 12. All of these nesting techniques display similar behavior: maxima at $500 \mathrm{~kW}$ system power and a slight drop in cost savings as system power increases. These results indicate that cost savings in excess of $15 \%$ may be possible with alternative channel nesting techniques, which could translate to savings of many tens of millions of dollars.

Though system mass savings of $6 \%$ are much less than what the early NHT work implied may be possible, these savings still have the potential for important mission impact. A $6 \%$ savings in mass for a 1-MW mission corresponds to approximately $800 \mathrm{~kg}$. This mass can either be left off, thus realizing the full drop in system cost predicted by the model, or re-purposed for more supplies or scientific instruments at the same launch cost. And because the cost savings associated with NHTs come from both launch costs and the shift to fewer thrusters, even if the mass savings is re-allocated to supplies or instruments, NHTs still provide a cost savings to the mission. This provides designers with increased flexibility as the full mission is being developed.

There are a number of features of NHTs that are not explicitly captured in this model, features whose addition would allow model users further insight when designing the propulsion string. One is the partial redundancy offered in NHTs: certain failure states may remove a single channel from operation, not the entire thruster, and a NHT with a wide throttling range such as the X3 would likely be able to throttle to a different condition to make up for the missing channel. Because the existing model only looks at redundancy from a string perspective, adding this feature with limited data on the types and relative frequencies of failure states seen on orbit would be difficult to do in such a way as to properly weight the cross-channel redundancy. However, as EP systems continue to proceed through flight qualification to flight, more of this information will become available. There is also footprint/area savings offered by NHTs over systems of many single-channel thrusters, a feature that would have an impact on spacecraft design. Additionally, further refinements to the cost model will be possible as more development work is done for NHTs and for high-power Hall thruster systems in general. These additional data may reveal scaling that differs from that proposed by the original work that may change the conclusions regarding propulsion string design. 


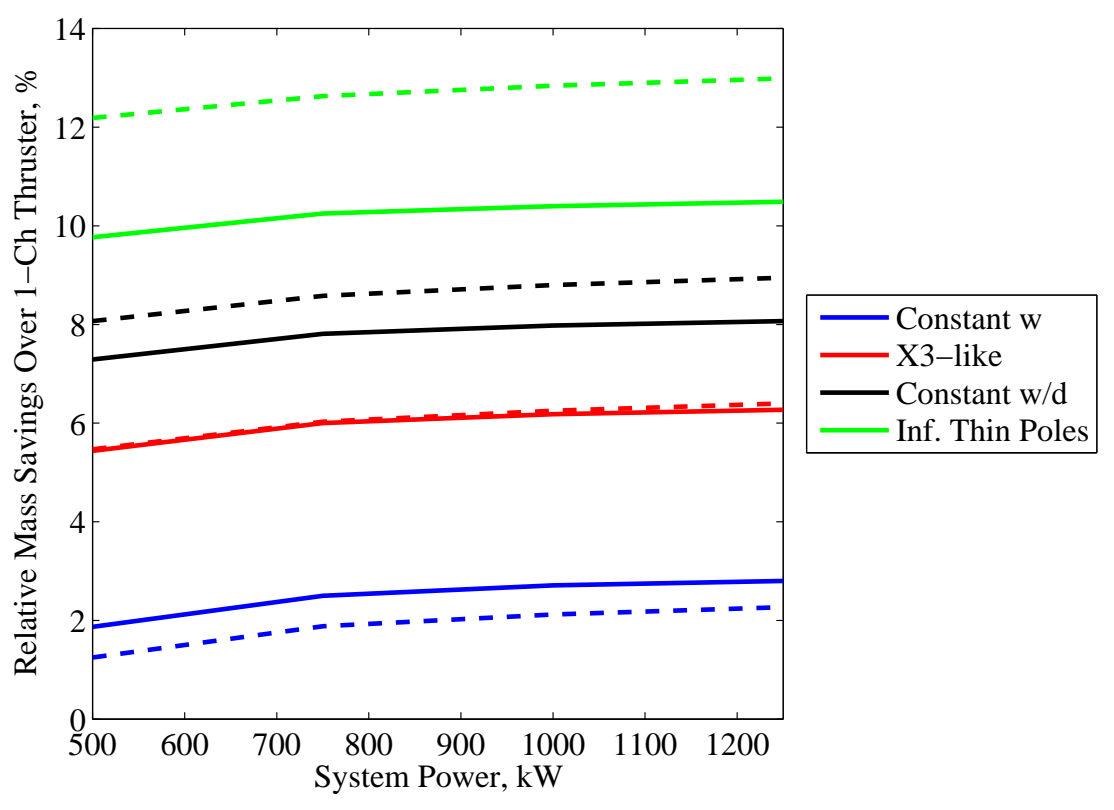

Figure 11. The relative mass savings provide by both 2-channel (solid line) and 3-channel (dashed line) configurations for all four example NHT nesting techniques. 


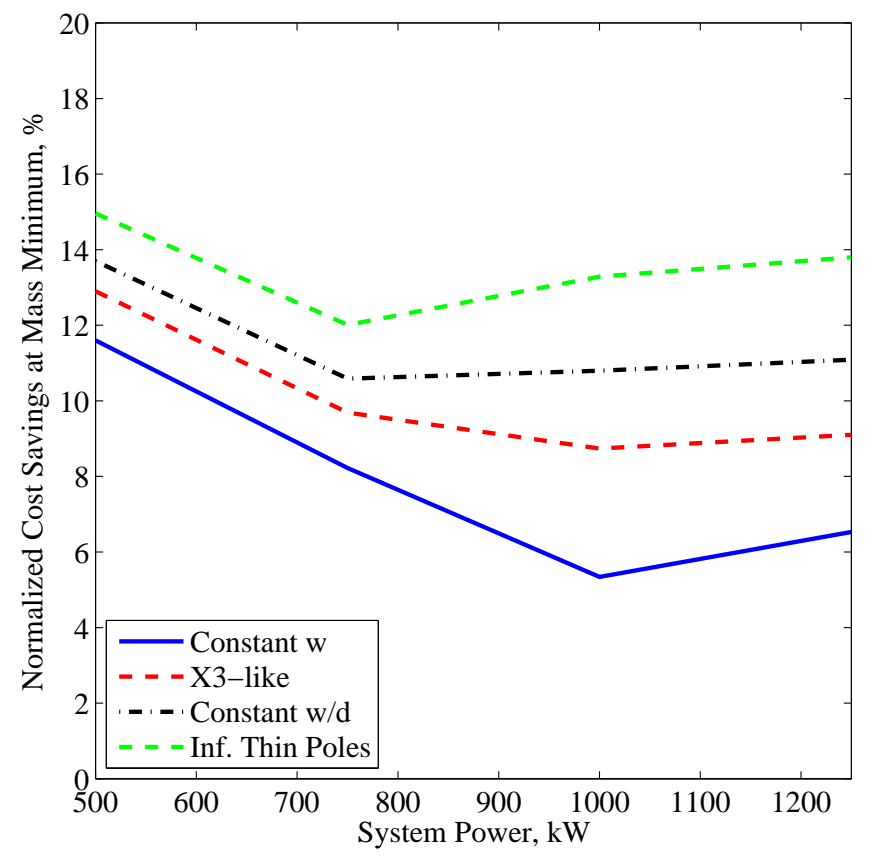

Figure 12. Cost savings at mass minimum versus system power level for all four example NHT nesting techniques.

\section{Conclusions}

Though there has been work in the literature to characterize the potential specific mass savings available by concentrically nesting the channels of Hall thrusters, no work to date has studied the effects at the system level. Determining the effects at this level is critical to the planning of future flight missions. To study these effects, we took an existing high-fidelity model for selecting EP string size and adapted it to capture NHTs. First, we developed an analytical expression for the thruster specific mass (kg mass per $\mathrm{kW}$ discharge power) for an arbitrary number of nested Hall thruster channels. We then evaluated this expression for a channel nesting technique based on a 3-channel NHT currently undergoing development work, the X3. This X3-like nesting was used as a baseline case to evaluate the potential impact of NHTs on propulsion system mass and cost. In addition to the modification to the thruster specific mass term, it was necessary to modify the XFC and cabling terms in the Hofer-Randolph mass model to account for the fact that these items are required once per channel in NHTs, not once per thruster.

Results for mission powers of $500 \mathrm{~kW}-1.25 \mathrm{MW}$ and a single redundant thruster indicate that for NHTs scaled like the X3, mass savings on the order of 4-7\% are available as compared to a system of single-channel thrusters. The mass savings rise with system power level. The physical reason for these savings is illuminated by plotting the results as a function of number of active channels instead of active thrusters: nesting the channels provides an increased packing efficiency. For a given number of active channels, the NHT geometry always provides improved mass for systems without redundant thrusters.

Adding redundant thrusters decreases the cost savings provided by NHTs. This is because redundant thrusters represent a large portion of system mass for systems of fewer thrusters. For systems of fewer than 10 active channels, the penalty incurred by even a single redundant thruster is enough to eliminate the mass savings offered by NHTs.

The Hofer-Randolph model also includes a cost portion, which we argue does not need modifications to represent NHTs within the intended accuracy of the model. The cost results for X3-like NHTs indicate that the modest 4-7\% mass savings translate to slightly improved cost savings of $9-13 \%$, which could represent several tens of millions of dollars. There are two contributions that NHTs make to reducing costs. First, 
the mass savings offered by NHTs translate directly to cost savings via launch costs. Second, NHTs provide mass minimums at smaller numbers of thrusters. Because the cost model shows strong minima, this serves to shift the mass-optimized cost smaller. These two effects combine to provide cost savings a few percentage points higher than the mass savings.

There are other potential techniques for nesting Hall thruster channels that may improve these savings. We developed three alternative nesting techniques and evaluated the thruster specific mass values of each, then applied these values to the mass and cost model. These results indicate that mass savings in excess of $8 \%$ may be possible, and that these mass savings correspond to cost savings in excess of $14 \%$.

At a system level, the savings offered by NHTs are relatively small. However, they still have the potential for significant spacecraft and mission planning impacts. It is clear that continuing to pursue NHT technology may result in further improvements to system mass and cost. NHTs have been shown to provide a lighter propulsion system and, by doing so with fewer thrusters, a less expensive one as well. NHTs will allow mission designers to better optimize their propulsion systems to minimize both mass and cost.

\section{Acknowledgments}

The authors gratefully acknowledge the helpful insight regarding Hall thruster design and scaling offered by Dr. Peter Peterson of NASA Glenn Research Center. Scott Hall is funded by a NASA Space Technology Research Fellowship under grant number NNX14AL67H and the guidance of NASA mentor Dr. Hani Kamhawi of NASA Glenn Research Center. Portions of the research described in this paper were carried out at the Jet Propulsion Laboratory, California Institute of Technology, under a contract with the National Aeronautics and Space Administration.

\section{References}

\footnotetext{
${ }^{1}$ Brophy, J. R., Gershman, R., Strange, N., Landau, D., Merrill, R., and Kerslake, T., "300-kW Solar Electric Propulsion System Configuration for Human Exploration of Near-Earth Asteroids," 47th Joint Propulsion Conference, Vol. 5514, AIAA, San Diego, CA, Aug. 2011.

${ }^{2}$ Strange, N., Merrill, R., Landau, D., Drake, B., Brophy, J., and Hofer, R., "Human missions to phobos and deimos using combined chemical and solar electric propulsion," 47th AIAA Joint Propulsion Conference, 2011.

${ }^{3}$ Merrill, R. G., Strange, N., Qu, M., and Hatten, N., "Mars conjunction crewed missions with a reusable hybrid architecture," Aerospace Conference, 2015 IEEE, IEEE, 2015, pp. 1-14.

${ }^{4}$ Gefert, L. P., Hack, K. J., and Kerslake, T. W., "Options for the human exploration of mars using solar electric propulsion," Space technology and applications international forum-1999, Vol. 458, AIP Publishing, 1999, pp. 1275-1280.

${ }^{5}$ Goebel, D. M. and Katz, I., Fundamentals of Electric Propulsion: Ion and Hall Thrusters, Vol. 1, John Wiley \& Sons, 2008.

${ }^{6}$ De Grys, K. H., Mathers, A., Welander, B., and Khayms, V., "Demonstration of 10,400 Hours of Operation on 4.5 kW Qualification Model Hall Thruster," 46th AIAA/ASME/SAE/ASEE Joint Propulsion Conference E Exhibit, Vol. 6698, Nashville, TN, July 2010.

${ }^{7}$ Herman, D. A., Santiago, W., Kamhawi, H., Polk, J. E., Snyder, J. S., Hofer, R. R., and Sekerak, M. J., "The Ion Propulsion System for the Asteroid Redirect Robotic Mission," 52nd AIAA/SAE/ASEE Joint Propulsion Conference, 2016, p. 4824 .

${ }^{8}$ Manzella, D., Jankovsky, R., and Hofer, R., "Laboratory Model 50 kW Hall Thruster," 38th AIAA Joint Propulsion Conference, American Institute of Aeronautics and Astronautics, Indianapolis, IN, July 2002.

${ }^{9}$ Hofer, R. R. and Randolph, T. M., "Mass and cost model for selecting thruster size in electric propulsion systems," Journal of Propulsion and Power, Vol. 29, No. 1, 2012, pp. 166-177.

${ }^{10}$ Grishin, S. D. and Leskov, L. V., Electrical Rocket Engines of Space Vehicles, Mashinostroenie, Moscow, 1989.

${ }^{11}$ Szabo, J., Pote, B., Hruby, V., Byrne, L., Tedrake, R., Kolencik, G., Kamhawi, H., and Haag, T. W., "A commercial one Newton Hall effect thruster for high power in-space missions," AIAA Paper, Vol. 6152, 2011.

${ }^{12}$ Liang, R. and Gallimore, A., "Performance of a Laboratory Hall Thruster with Two Concentric Discharge Channels," 2010.

${ }^{13}$ Liang, R. and Gallimore, A. D., "Far-Field Plume Measurements of a Nested-Channel Hall-Effect Thruster," 49th AIAA Aerospace Sciences Meeting, American Institute of Aeronautics and Astronautics, Orlando, FL, Jan. 2011.

${ }^{14}$ Liang, R., The Combination of Two Concentric Discharge Channels into a Nested Hall-Effect Thruster, Ph.D. thesis, University of Michigan, Ann Arbor, MI, 2013.

${ }^{15}$ Florenz, R. E., Gallimore, A. D., and Peterson, P., "Developmental Status of a 100-kW Class Laboratory Nested Channel Hall Thruster," 32nd International Electric Propulsion Conference, Wiesbaden, Germany, Sept. 2011.

${ }^{16}$ Florenz, R. E., The X3 100-kW Class Nested-Channel Hall Thruster: Motivation, Implementation, and Initial Performance, Ph.D. thesis, University of Michigan, Ann Arbor, MI, 2013.

${ }^{17}$ Hall, S. J., Florenz, R. E., Gallimore, A. D., Kamhawi, H., Brown, D. L., Polk, J. E., Goebel, D., and Hofer, R. R., "Im-
} 
plementation and Initial Validation of a 100-kW Class Nested-channel Hall Thruster," 50th AIAA Joint Propulsion Conference, American Institute of Aeronautics and Astronautics, Cleveland, OH, July 2014.

${ }^{18}$ Hall, S. J., Cusson, S. E., and Gallimore, A. D., "30-kW Performance of a 100-kW Class Nested-channel Hall Thruster," 34th International Electric Propulsion Conference, Kobe-Hyogo, Japan, July 2015.

${ }^{19}$ Huang, W., Shastry, R., Soulas, G. C., and Kamhawi, H., "Farfield Plume Measurement and Analysis on the NASA-300M and NASA-300MS," 33rd International Electric Propulsion Conference, Washington D.C., 2013.

${ }^{20}$ Brown, D. L., Haas, J. M., Peterson, P. Y., and Kirtley, D. E., "Development of High-Power Electric Propulsion Technology for Near-Term and Mid-Term Space Power," 57th Joint Army Navy NASA Air Force Propulsion Meeting, Colorado Springs, CO, May 2010.

${ }^{21}$ Brophy, J. R., Marcucci, M. G., Ganapathi, G. B., Garner, C. E., Henry, M. D., Nakazono, B., and Noon, D., "The ion propulsion system for Dawn," AIAA Paper, Vol. 4542, 2003.

${ }^{22}$ Manzella, D., Scaling Hall Thrusters to High Power, Ph.D. thesis, Stanford University, Stanford, CA, 2005.

${ }^{23}$ Dannenmayer, K. and Mazouffre, S., "Elementary scaling relations for Hall effect thrusters," Journal of Propulsion and Power, Vol. 27, No. 1, 2011, pp. 236-245. 